\title{
Using population viability analysis to guide research and conservation actions for Australia's threatened malleefowl Leipoa ocellata
}

\author{
MichaEL Bode and KARL E. C. BRENNAN
}

\begin{abstract}
Malleefowl Leipoa ocellata populations across Australia are declining and the range of the species is contracting. Despite a century of research much uncertainty remains about which factors are driving this decline. Consequently, it is also unclear which conservation actions will reduce the species' extinction risk. In particular, we lack a quantitative understanding of malleefowl population dynamics. Here we use estimates derived from the literature to provide the first parametrization of a population viability analysis (PVA) for malleefowl. This model creates a quantitative framework for synthesizing existing information and comparing potential management strategies, and will help guide research activities by identifying critical aspects of the malleefowl's life history. We model population dynamics as stochastic events that depend on individual characteristics, weather conditions and local management actions. Our PVA indicates that an isolated population of 32 adult birds would almost certainly decline to extinction over a 20-year period. Translocating and releasing captive-bred juveniles slows this rate of decline and intensively baiting for foxes can reverse it. Adult mortality rates have the greatest influence on population viability, and land managers should therefore prioritize conservation actions that target adult survivorship over actions that benefit earlier life stages. Quantitative research on the malleefowl should focus on the demographics of the adult life stage, their dispersal and the impacts of fire and grazing. Our analysis highlights the role of PVA models in assessing the cost-effectiveness of alternative management actions, and framing future research priorities for threatened species.
\end{abstract}

Keywords 1080 baiting, Australia, decision theory, Leipoa ocellata, malleefowl, mound builders, return on investment, Vulpes vulpes

This paper contains supplementary material that can be found online at http://journals.cambridge.org.

Michael Bode (Corresponding author) School of Botany, University of Melbourne, Parkville, VIC 3010, Australia. E-mail mbode@unimelb.edu.au

Karl E.C. Brennan Goldfields Region, Department of Environment \& Conservation, Kalgoorlie, Australia

Received 21 October 2010. Revision requested 19 January 2011.

Accepted 15 March 2011.

\section{Introduction}

alleefowl Leipoa ocellata are large (adults $1.8-2.2 \mathrm{~kg}$ ), 1 mound-building birds that were historically distributed throughout southern and central Australia's arid and semi-arid woodlands and shrublands (Department of the Environment, 2010). Despite their relatively low international profile, the species holds iconic status within the Australian conservation community. The attention is a response to the species' large size, distinctive mounds and complex reproductive behaviour. Conservation management of the malleefowl dates from at least the early 20 th century, when an early translocation attempt saw a population briefly established on fox-free Kangaroo Island, South Australia (Mellor, 1911; North, 1917). The species is the focus of many non-government conservation groups (e.g. the Victorian Malleefowl Recovery Group, and the Malleefowl Preservation Group), and successfully attracts considerable research, monitoring and management funding. In particular, there is a multi-regional community-based monitoring programme focused on malleefowl, which annually records the number of active mounds at over 60 sites across southern Australia (Benshemesh, 1997, 2004). Monitoring and managing malleefowl is also a licensing requirement of some mining operations (e.g. iron ore mines in Western Australia's Goldfields region).

In addition to ongoing population monitoring, for the last 60 years ornithological researchers have closely investigated many aspects of the malleefowl's life-history. Following Frith's pioneering research on the complex structure and maintenance of reproductive mounds (Frith, 1959), studies have explored the species' habitat preferences (Parsons, 2008, 2009), reproduction (Brickhill, 1987), fecundity (Priddel \& Wheeler, 2005), and juvenile survivorship (Priddel \& Wheeler, 1989, 1996). However, our understanding of the species remains fragmented; there is much uncertainty about how its behaviours and life-history characteristics interact to determine population trends. Consequently, the relative importance of the different malleefowl life history attributes remains unquantified. The question of primary conservation concern-how to cost-effectively ensure viable malleefowl populations in the face of ongoing and novel threats-is therefore uncertain. To address this issue effectively we must first consolidate the wealth of existing malleefowl research into a coherent quantitative model of population dynamics. This is our primary aim here. 
The demand for an accurate, quantitative understanding of malleefowl population dynamics is urgent, as recent studies reveal a species in rapid decline but for unclear reasons (Benshemesh et al., 2007). Malleefowl are categorized as threatened across their entire range by state and federal governments, and are listed as Vulnerable on the IUCN Red List (BirdLife International, 2008; Department of the Environment, 2010). Despite their large population (c. 100,000) and broad range $\left(1,420,000 \mathrm{~km}^{2}\right.$; BirdLife International, 2010), the species faces a suite of potentially threatening processes, including the degradation of habitat, introduced mammalian predators, competition with introduced grazers, and the alteration of historical fire regimes. However, it is not clear which individual process or combination of processes bears primary responsibility for the ongoing decline of the species (Benshemesh et al., 2007). Foxes Vulpes vulpes have long been considered the principal threat (Mellor, 1911; Wheeler \& Priddel, 2009), as the bird falls within the 'critical weight range' (Chisholm \& Taylor, 2010) of species driven to extinction by fox predation. To complicate feral predator management, however, feral cats Felis catus also prey on chicks (Wheeler \& Priddel, 2009). As an alternative to this top-down orthodoxy, some researchers argue that grazing by sheep and rabbits has greater impact on malleefowl populations than feral predators. Sheep density is a significant predictor of malleefowl absence (Parsons, 2008), and comparative studies suggest that grazing could reduce the density of malleefowl by a factor of 10 (Frith, 1962). A recent analysis of long-term trend data revealed equivocal evidence about the efficacy of fox baiting on malleefowl population trends (Benshemesh et al., 2007). This is of concern given that fox baiting is being applied at considerable expense to help conserve malleefowl populations (Benshemesh et al., 2007; BirdLife International, 2010).

Here we describe a population viability analysis (PVA) model for malleefowl. The process of constructing this model involves a review and synthesis of existing quantitative information on malleefowl population dynamics. A quantitative PVA is a valuable decision-support tool for conservation managers, helping them to make informed predictions about the future of populations (Beissinger \& McCullough, 2002). In addition, a PVA model provides a process-based link between threatening processes, management actions and the viability of malleefowl populations. This allows management choices to be made on the basis of a transparent description of malleefowl dynamics, rather than expert opinion, which is difficult to reproduce or defend (Pullin \& Knight, 2009). The explicit and systematic process required to construct a PVA model helps to identify aspects of malleefowl population dynamics that are not currently well understood, in a manner relevant to making predictions. This helps to focus data collection on weak points in our understanding of the species. PVAs also enable sensitivity analysis of life history characteristics. These help to identify which parameters disproportionately affect population viability and may therefore need to be estimated more accurately (Buckley et al., 2003). Finally, PVA models facilitate an examination of the cost-effectiveness of alternative management actions. We focus this analysis on two management interventions currently applied to malleefowl populations: fox baiting and translocation following captive breeding (Benshemesh, 2007; Gillespie, 2007).

\section{Methods}

\section{Model structure}

We model the dynamics of malleefowl populations using a stochastic, age-structured model that simulates a single, non-spatial population. The simplifications involved in this PVA model mean that, although it can offer qualified insights into management decision-making for threatened species its ability to make quantitative predictions about specific populations is limited. There are several motivations for this model formulation. Firstly, its purpose is to summarize our existing quantitative knowledge of malleefowl population dynamics, and a straightforward model provides a more transparent perspective. Secondly, the PVA cannot closely reflect a given set of local conditions, given that our parametrization uses data sourced from a wide range of localities. Thirdly, this is the first processbased model of malleefowl population dynamics and a more general, preliminary approach is therefore appropriate. This non-spatial model will provide the groundwork for the more realistic, site-specific PVA models that managers require to make predictions about specific populations. In the following sections we discuss the main elements of the model. The Appendix provides detailed information on model parameters.

\section{Stage classes}

The model follows malleefowl individuals from the laying of each egg through to their eventual senescence at 25 years (Benshemesh, 2007; BirdLife International, 2010). Malleefowl face significant mortality risk throughout their lives, and therefore few individuals reach this upper age limit. We iterate the model in seasonal (i.e. 3-month) time steps and, although we model the exact age of each individual, we group them into five life-stages: eggs ( $0-3$ months), hatchlings (3-6 months), juveniles (6-9 months), subadults ( 9 months to 2 years) and adults ( $>2$ years). Malleefowl demographics undoubtedly vary within these five life stages. Fecundity, for example, almost certainly changes as an adult ages. Our choice of resolution was driven by the published data available for parametrization. We anticipate that the number of life stages in the model would increase as more detailed demographic data become available. 


\section{Mortality}

The mortality of individual malleefowl at each time step is a stochastic event that depends on age, the environment and the fox control regime. We calculate the fate of each individual probabilistically (Appendix). Researchers have investigated the influence of foxes on the survival of hatchlings and juveniles (Priddel \& Wheeler, 1997), allowing us to estimate mortality rates during each of the first three life-stages in both the presence and absence of fox baiting. Mortality rates for adult malleefowl are not available despite recognition that adult longevity contributes disproportionately to persistence of the species. We therefore estimate the mortality of subadults and adults using trapping time-series data (Priddel \& Wheeler, 2003). Although there are reports of adults being killed by foxes (Booth, 1987), most studies have shown low or non-existent levels of predation on adults (Benshemesh, 1994). Furthermore, adult mortality rates with and without fox control have not been calculated. We therefore treat mortality during the adult stage as unaffected by fox control efforts. For similar reasons we do not include additional mortality effects of cat populations at any life stage.

During the egg stage survival also depends on the weather. After intense rainfall mounds can be inundated and the developing embryos perish. Brickhill (1987) observed this during the summer of 1984 near Griffith, New South Wales, after 24 hours with $>100 \mathrm{~mm}$ of rain. Based on local historical records we calculate the annual probability of such an event. Although annual rainfall and the materials used to construct mounds vary across the malleefowl's range, we assume that this value applies to broader contexts. We recognize that there are limitations to drawing values from anecdotal observations and we discuss these limitations later.

\section{Reproductive behaviour and fecundity}

Adults reach breeding age after 2 years, when they attempt to form monogamous pair-bonds that persist until one individual dies (Frith, 1959). Even in the presence of additional unpaired adults of reproductive age, polygamous behaviour is rare and may occur only under unusual weather conditions (Weathers et al., 1990). If an unattached replacement mate is not available a single adult will remain unpaired in the population until a mate becomes available through either recruitment or mortality. Egg laying begins in late September, with an egg laid on average every 6.4 days (Priddel \& Wheeler, 2005). Mean observed clutch size is 16.6 eggs per pair (Appendix). Insufficient winter rainfall is known to delay or even cancel the onset of laying (Priddel \& Wheeler, 2005) but the critical minimum rainfall threshold is unknown. There is anecdotal evidence that a population of $\mathrm{c}$. six breeding pairs of malleefowl near Windarling, Western Australia, did not breed during a severe drought in 2007, when only $10.5 \mathrm{~mm}$ of winter rainfall was recorded (M. Bamford, pers. comm.). Comparison with 100 years of historical rainfall data show that such conditions occur in the Windarling region once every 85 years.

\section{Initial conditions}

We begin the viability simulations with a population structure resembling the population surveyed in 1999 at Yalgogrin, New South Wales (Priddel \& Wheeler, 2003). This population contained 32 paired and unpaired breeding adults with a mean age of 6.4 years, from new recruits to 11 year-old adults. Our initial simulations had 32 individuals with ages drawn from a uniform distribution between 2 and 11. Priddel \& Wheeler (2003) did not count the number of juveniles but the population received a mean of 1.4 new recruits into the breeding adult population each year (14 recruits observed over a decade). Given the expected rates of pre-adult survivorship, this recruitment implies the additional, unobserved existence of 24 hatchlings and four juveniles. We included these malleefowl in our initial population. Each simulation begins in a season that was selected at random.

\section{Measuring population viability}

We model population trends over a period of 20 years, longer than the mean expected lifetime of a malleefowl (Benshemesh, 2007). Pre-breeding mortality in malleefowl is naturally high (Frith, 1962; Benshemesh, 2007) and the variation in total population size (i.e. the sum of all individuals in the population) will therefore be driven by fluctuations in the number of non-adults. Adult recruitment and turnover are infrequent events. The long-term viability of a population thus relates more closely to the number of adults than to the total population size. Estimation of the number of active nests (a proxy for the number of adults in the population) is also the metric used to monitor malleefowl dynamics. We therefore monitor the change in the adult population through time as a metric of population viability. Both demographic and environmental stochasticity affect the trajectory of the malleefowl population and we consequently base our viability estimates on the mean of 1,000 simulations, reporting the $95 \%$ confidence intervals.

\section{Sensitivity analyses}

Many of the model parameters (Appendix) are subject to considerable uncertainty. We sourced most from smallscale studies in specific regions, and their applicability across the malleefowl's broad range is unproven. Despite this, they provide a useful set of estimates for a standard simulation-based sensitivity analysis (Caswell, 2001) on the four key malleefowl life-history parameters: (1) egg mortality, which is equivalent to adult fecundity, (2) hatchling mortality, 
(3) juvenile mortality, and (4) adult mortality. We decreased each of the mortality probabilities by 0.05 from its nominal value and then calculated the effect of this alteration on 10 -year trends in the adult population (based on the mean of 1, ooo simulations).

Climate affects the modelled dynamics of malleefowl via two parameters: drought-suspended reproduction and egg mortality during high-rainfall events. We examine the sensitivity of long-term malleefowl population trajectories to changes in these parameters by decreasing the probability of each event by $50 \%$. We further consider the impact of increasing the rate of these environmental extremes by calculating the population viability in scenarios where each increases five-fold. Both sensitivities are assessed over 10 years.

\section{Cost-effectiveness of alternate management strategies}

To demonstrate how PVA models could inform costeffective management decisions we contrast the relative utility of three alternative conservation strategies for an isolated malleefowl population. We consider intensive fox control (bait), the translocation and release of captive-bred malleefowl juveniles (release), and intensive fox control augmented by the release of malleefowl hatchlings sourced from a captive population (bait and release; Benshemesh, 2007). The first will reduce the mortality of malleefowl eggs and chicks, the second will effectively increase fecundity, and the third will do both (at a greater cost). To facilitate comparisons we equalize the cost of the bait option and the release option as follows.

We assume that the baiting strategy required for our simulated population mirrors that in the study which provided our estimates of the effects of fox baiting (Priddel \& Wheeler, 1997): both the region occupied by malleefowl ( 558 ha of remnant native vegetation) and a 6,842 ha buffer zone were baited. Sodium monofluoroacetate (compound 1080) baits were placed fortnightly at a high density (averaging one bait per $13 \mathrm{ha}$ ). We estimate the cost of distributing baits at AUD 0.63 each (2010 prices), comprising AUD 0.25 per bait and AUD 0.38 per delivery (Warburton \& Cullen, 1995; Department of Agriculture, 2010). The estimated annual cost of intensive fox control is thus AUD 8,064. In the release strategy managers maintain captive populations of malleefowl and release offspring into the wild, supplementing natural recruits. Malleefowl are relatively easy to rear (Gillespie, 2007) and have been successfully bred in Australian zoos. Threatened bird species have been raised in captivity for c. AUD 3.5 per bird per day (McCutcheon, 2009) and malleefowl are typically released into the wild after 10 months (Gillespie, 2007). With an annual budget equal to the AUD 8,064 required for fox baiting, managers could release seven malleefowl juveniles into the population each year.
We ran 1,00o simulations of our malleefowl population model for each of the three management actions, and contrast the expected viability of the resultant malleefowl populations after 10 years. Because bait and release is a more expensive strategy we measure its relative benefit by considering a two-population management scenario. With the same budget, managers can either choose to bait and release in one population and take no action in the other, bait two populations, or release in two populations.

\section{Results}

\section{Measuring viability}

Given the model structure and parameter estimates described, unmanaged and isolated malleefowl populations exhibit an inexorable decline over 20 years (Fig. 1), with the average final population diminished by $99 \%$ ( $95 \%$ confidence interval, CI, $98-100 \%$ decrease, minimum decrease $=96 \%$ ). The PVA model reveals isolated populations that cannot produce sufficient new recruits to offset mortality. Our current understanding of malleefowl dynamics thus shows a population in decline towards local extinction.

\section{Sensitivity analyses}

The life history parameter with the most impact on population viability was the probability of adult mortality. A reduction of 0.05 in this probability improved the viability of the average malleefowl population by $8 \%$ (i.e. the 10 -year population decrease ranged from 96 to $88 \%$ ). Varying each mortality parameter by $5 \%$ yielded the same result. In contrast, reductions of 0.05 in egg, hatchling or juvenile mortality probabilities had little impact on the mean population viability, improving it by only 1,3 and $1 \%$, respectively.

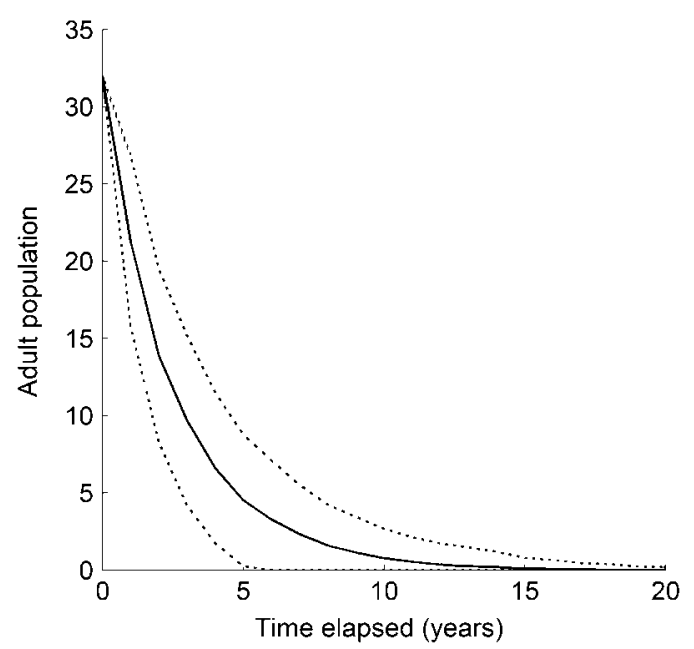

FIG. 1 Average malleefowl Leipoa ocellata population trajectory (solid line) in the PVA model, showing number of adults over 20 years, without management. Solid line is the mean of 1,000 simulations; dashed lines denote $95 \%$ confidence intervals. 
Decreasing the frequency of extreme rainfall events reduced the magnitude of the 10 -year population decrease by $<1 \%$. Even drastic, five-fold increases in the frequency of these events had very little effect on rates of population decrease. These results were subject to the stochastic variation inherent in the PVA model; Fig. 2 shows the viability distributions resulting from each of the parameters analysed.

\section{Cost-effectiveness of management strategies}

Fig. 3 compares the viability of malleefowl populations when managers undertake the three interventions. Compared with the 10-year decrease of $96 \%$ ( $95 \%$ CI $88-100 \%$ decrease) in the absence of intervention, each of the management strategies is expected to improve the population's viability. Fox baiting alone results in a population that increases by an average of $39 \%$ over 10 years (95\% CI $162 \%$ increase $-56 \%$ decrease). With the same budget, the release of captive-bred juveniles could not reverse the population decline, although the 10-year decrease was reduced to $62 \%$ (95\% CI $40 \%$ decrease- $81 \%$ decrease). Taking both management actions simultaneously results in a population that increases by an average of $85 \%$ over 10 years (95\% CI 190\% increase- $13 \%$ decrease). Compared with baiting alone, bait and release achieves approximately double the rate of increase for twice the cost. Because of demographic and environmental stochasticity none of the management options can guarantee that a population exhibits positive net growth over the 10-year period, although bait and release only results in a declining population in $2 \%$ of simulations. Baiting alone results in population decline in $16 \%$ of cases; when captive breeding was the only intervention all of the simulations exhibited declines.

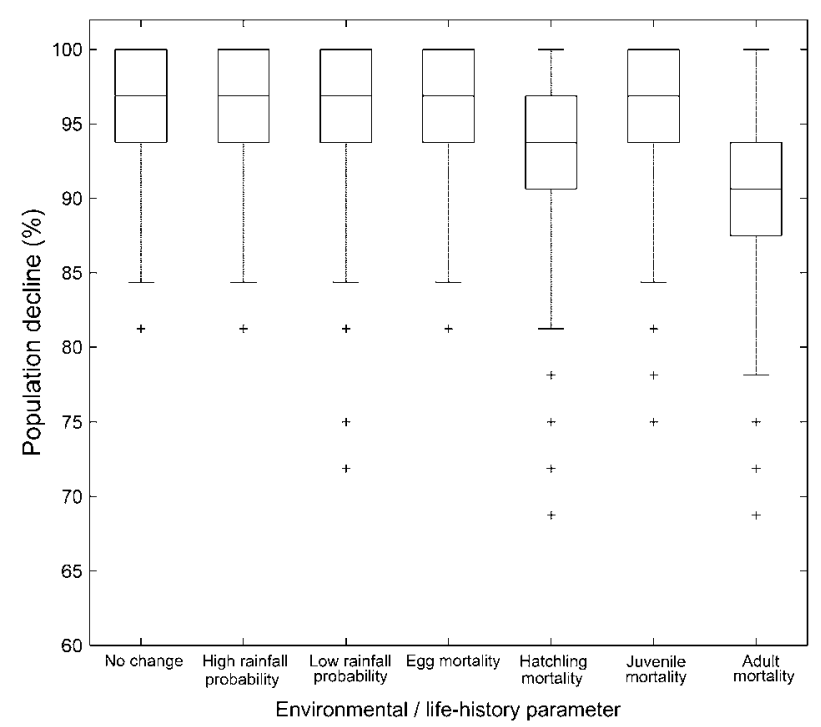

FIG. 2 Sensitivity of malleefowl population viability to a reduction of 0.05 in the two rainfall probabilities and four mortality probabilities compared to no change. Box and whisker plots indicate the percentage decrease over 10 years in 1,000 stochastic simulations.

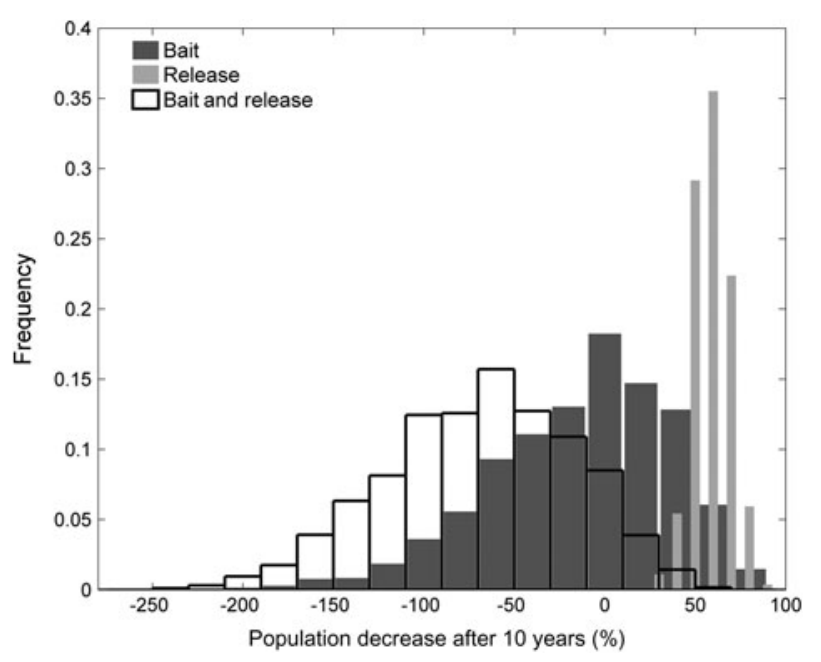

FIG. 3 Decreases of malleefowl population over 10 years if managers implement intensive fox baiting, release captive-bred juveniles, or both. Frequency distributions are the results of 1,00o simulations.

Because bait and release is a more expensive management strategy, a two-population scenario facilitates a constantbudget comparison of bait and release. If managers spend a budget of AUD 6,128 on bait and release in population one while implementing no management in population two, the possible 10-year outcomes are, with associated probabilities: no increasing populations ( $2 \%)$, one increasing population (98\%), and two increasing populations (o\%). Alternatively, managers can bait both populations with the same budget, with the possible outcomes being: no increasing populations (2.6\%), one increasing population (26.9\%), and two increasing populations $(70.5 \%)$. If managers release in both populations without baiting, neither will increase.

\section{Discussion}

Our PVA model of malleefowl offers a demographicallyfocused perspective on the viability of this threatened species and shows that the conservation situation for the species is not robust. This conclusion is unsurprising and concurs with nationwide surveys of malleefowl abundance over the last 30 years that show widespread declines in local populations and drastic range contractions (Benshemesh et al., 2007). Based on our best quantitative estimates of malleefowl population dynamics, a small isolated population of 32 malleefowl will decline to extinction within the next 20 years unless there is management intervention. Many of our parameter estimates are uncertain, however, and this conclusion may be overly pessimistic. It is important to note that many of our data were sourced from analyses of malleefowl populations in pockets of remnant native vegetation surrounded by ecologically degraded agricultural land (Priddel \& Wheeler, 2003, 2005). As a result, these dynamics may not accurately reflect the prospects of malleefowl populations in less degraded areas such as the continuous eucalypt forests of 
Western Australia's Great Western Woodlands. However, the best quality habitat for malleefowl is in high rainfall areas with better quality soils, which are the areas where clearance for agriculture has occurred (Frith, 1962; Benshemesh, 1992). Even areas remaining uncleared within these regions are often degraded (Parsons, 2009).

A PVA model also allows an appraisal of our current knowledge about the species' ecology and dynamics. Our review of the literature reveals that quantitative research has been unevenly distributed across the malleefowl's life history. Although some parameters have been measured quantitatively and repeatedly (e.g. the number of eggs laid in a mound) many had to be derived from data that was gathered for other purposes, and the results will thus contain a substantial degree of uncertainty. Unfortunately, if the research distribution is compared with the sensitivity of the different life-history stages (Fig. 2), it is clear that the most important life-history attributes are also the least investigated. In particular, research attention has focused on reproduction and survivorship of eggs and chicks, rather than the adult life stage. Of the research that did focus on adults, most was concerned with their reproductive behaviour (and thus indirectly the early life stages), and primarily considered behavioural issues such as the dynamic use of nesting mounds. This bias presumably reflects a natural history focus on the species' unusual nesting behaviour. Our unbalanced understanding of malleefowl-a combination of intense research into some parameters and little information on the remainder-may be common in the conservation of iconic threatened species. Logistic factors may have exacerbated the disproportionate focus on juveniles: the dynamics of the adult stage occur over longer periods and are more difficult and expensive to measure. However, the distribution of monitoring effort should reflect the management value of the information it will produce, not simply the cost of acquiring that information (Runge et al., 2011).

This lack of data casts doubt on our ability to make accurate predictions about the species. For example, we estimated that c. $10 \%$ of adults suffer mortality each year, a level that resulted in non-viable malleefowl populations. However, if our parameter overestimated the annual probability of adult mortality by 0.08 , an isolated malleefowl population could persist (i.e. the $95 \% \mathrm{CI}$ of population decline would overlap zero). Given the sparse data used to construct our estimate of adult survivorship (a single 10-year study), this amount of error would not be surprising. Similarly, if adult mortality is reduced by fox baiting, the predicted performance of this intervention could improve markedly, as evidenced by the sensitivity of the adult mortality parameter (Fig. 2). Resolution of demographic parameters in the later life stages should therefore be a priority for future research.

This lack of information extends to our understanding of how the environment affects malleefowl dynamics. There is good quantitative data showing that amount of rainfall between May and December affects the length of the laying period and thus the number of eggs laid (Priddel \& Wheeler, 2005). We also know that heavy inundations can exert marked effects on reproduction but this understanding is based only on anecdotal evidence. Generally, a researcher has noted a particularly extreme weather event interfering with normal malleefowl dynamics. For example, high summer rainfall affects egg viability and low winter rainfall interrupts laying. Thus, we can only infer that more extreme weather events are likely to elicit the same response. A more nuanced understanding is required for the purposes of prediction but the high variability of Australia's arid environments will make such parametrizations expensive. Given the low sensitivity of model predictions to parameters concerning the egg survivorship, research on the effect of climate should focus on the adult life stages, rather than fecundity or egg mortality.

\section{PVA model structure}

The structure of a useful PVA model is a delicate balance of known and unknown factors. Clearly, the model is constrained by the availability of ecological data and the set of observed processes. For example, malleefowl dispersal has rarely been observed (but see van der Waag, 2007, and Coombes et al., 2007, for exceptions over short distances), and the rate of long-distance dispersal between separate local populations is unknown. It is difficult, therefore, to incorporate into our PVA issues such as potential rescue effects (Gotelli, 1991), or source-sink dynamics (Pulliam, 1988). On the other hand, the inclusion of processes with less uncertainty can allow managers to estimate the importance of these processes for population viability. Foxes have been observed to prey on malleefowl eggs (Frith, 1959), for example, and including egg predation at the estimated rate allows its impact on population viability to be assessed, and to determine whether efforts to reduce egg predation alone would significantly benefit malleefowl populations.

Of the environmental processes that we did not consider in the model, the three most critical are habitat loss, fire and the effects of grazing. Although habitat loss has historically been the primary driver of malleefowl declines, recent changes to land clearing rights on private land have greatly reduced this threat in the main malleefowl states (Victoria, New South Wales, South Australia and Western Australia; Benshemesh, 2007). The main effect of habitat loss will be to reduce the carrying capacity of a population, and this should therefore be included as required by specific scenarios. Fire affects malleefowl populations immediately, probably through direct mortality, and perhaps in the longer term through decreased reproduction (Benshemesh, 1992). We chose not to include fire as there is almost no quantitative information available to attempt to parametrize the model. It is not clear, for example, how much of the immediate decline in adult abundance is the result of direct 
mortality and how much is the result of temporary emigration. Although these two processes will have the same immediate effect on adult abundance in the local population, they will result in different recovery trajectories. We also do not know the rate at which suitable malleefowl habitat recovers following fire and how any recovery affects malleefowl population parameters. Estimated recovery times range between 5 and 80 years (Woinarski, 1989; Benshemesh, 1992, 1997, 2008; Ayers et al., 1996). Given that fire may be an important determinant of population dynamics (particularly given its impact on adults), malleefowl conservation would benefit from improved quantitative information on precisely how fire perturbs the species, and how populations and habitat recover. Grazing has been identified as an important anthropogenic driver of malleefowl population dynamics and the removal of exotic competitors has been suggested as a management action (Frith, 1962; Benshemesh, 2007). The reduction or exclusion of livestock is a management action practised in a wide range of Australian conservation contexts, and assigning costs to the approach would thus be relatively straightforward. Unfortunately, a lack of evidence quantifying the extent of the benefit, and the process by which this benefit is realized, prevents its inclusion in the PVA.

A further omission from our model was the potential benefit of controlling feral cat populations. While cats prey on malleefowl chicks (Wheeler \& Priddel, 2009), to date no study has compared quantitatively the degree to which malleefowl benefit from feral cat control. This is not surprising as feral cat control poses a formidable challenge. Cats seldom take baits except during time of prey shortages and even then specially formulated baits are required. We suspect that the use of baits that are attractive to both cats and foxes could increase the effectiveness of feral predator control but the lack of data prohibits addressing this question with the model.

\section{Management implications}

The results of our sensitivity analyses (Fig. 2) have management implications (Buckley et al., 2007), identifying which life-history events managers should target to ensure the greatest positive effect on population viability (assuming that our best estimates are accurate). They indicate the greater relative importance of older malleefowl, a result that agrees with an intuitive understanding of fecund, long-lived species in highly variable environments such as Australia's arid zone. In addition to supporting this understanding, a PVA quantitatively measures the increased value of later life stages, which helps determine the relative value of alternative conservation interventions. For example, road collisions can kill substantial numbers of adult malleefowl: Benshemesh (2007) reported 13 malleefowl mortalities along a $2-\mathrm{km}$ stretch of road. The erection of road signs has reduced wildlife-vehicle collisions by $50 \%$ in other contexts at low cost (Sullivan et al., 2004). It therefore appears likely that this management strategy would represent an effective use of resources. The National Recovery Plan for malleefowl (Benshemesh, 2007) identifies, in order of priority, six key threats that management should focus on: reduce habitat loss, reduce grazing pressure, reduce fire threats, reduce predation, reduce population isolation, promote malleefowl-friendly agricultural practices, and reduce mortality on roads. If it were possible to quantify the expected effect of these interventions it would be possible to rank these priorities in a transparent manner.

The sensitivity analyses do not identify climatic conditions as key determinants of malleefowl population dynamics (Fig. 2). Decreasing the probability of high and low rainfall events by $50 \%$ has no discernible effect on the rate of population decline. Even increasing the frequency of extreme climatic events five-fold affects population decline rates by $<1 \%$. This result is consistent with the low sensitivity of the egg mortality parameter, as in our PVA rainfall affects the egg stage (either the number laid or the proportion that survive to hatching). It therefore appears unlikely that more frequent climatic extremes will seriously affect malleefowl population trends if the effect of these events is limited to the egg stage. Other analyses that have shown climate change detrimentally affecting malleefowl have been based on broad-scale bioclimatic changes (e.g. a reduction in malleefowl habitat; Brereton et al., 1995), which we have not considered here. Alternatively, environmental changes could impact malleefowl population dynamics if they affect survivorship during the sensitive adult life stage.

The indication that intensive fox baiting can improve the viability of malleefowl populations provides an illustration of how a simulation approach can provide insight into trends that are difficult to measure empirically. Firstly, in highly variable arid environments it can be difficult to separate empirically the benefits of a management action from the background environmental variability. Much of the high quality malleefowl data are collected from regions that have recently been experiencing drought conditions. Repeated simulations with different environmental conditions can offer insight into whether observed trends are attributable to transient environmental conditions, or underlying deficits in the population dynamics. Secondly, if malleefowl populations were declining regardless of fox presence it would be difficult to prove the utility of baiting because doing so would require distinguishing two different decline rates. This is particularly true when the immediate benefits accrue to juvenile malleefowl, which are not counted by survey methods that focus on active nesting mounds.

The support offered for fox baiting in these analyses comes with strong caveats. Our results reflect an intense baiting strategy with a broad buffer region around the population (Priddel \& Wheeler, 1997). The benefits we predict are unlikely to result from smaller-scale actions that 
only bait sporadically; this difference explains at least part of the discrepancy between our results and the metaanalysis described in Benshemesh et al. (2007). There are also negative outcomes associated with fox baiting that we did not consider. Firstly, it would be very expensive to extend this level of fox baiting over a significant proportion of the malleefowl distribution range. Secondly, a lack of resistance in native species constrains the use of compound 1080 in South Australia, New South Wales and Victoria (these are large parts of the malleefowl range). Thirdly, by negatively affecting the populations of apex predators (Kortner \& Watson, 2005) 1080 baiting can have potentially detrimental indirect effects on native wildlife through mesopredator release (Glen \& Dickman, 2005).

The comparison between alternative management strategies illustrates how PVA models can rapidly provide information on the contrasting benefits of malleefowl management alternatives. For instance, releasing captive-bred malleefowl juveniles is clearly not a cost-effective management strategy on its own but, if used in conjunction with fox baiting, can augment the expected population growth rate (Fig. 3). Translocation policies at the state, government and international level all require the mitigation of threatening processes before translocation is undertaken; these results provide support for this precondition. Alternatively, the same budget could be spent baiting two separate populations, spreading the risk of an overall population decline.

\section{Conclusion}

Little is known about most of the earth's threatened species: many are identified only by a museum specimen, a taxonomic description and perhaps a handful of occurrence locations. Our ability to parametrize a PVA model of malleefowl attests to the intensive efforts of researchers, particularly over the last 2 decades. This model reflects how much we know about malleefowl dynamics; the fact that we can begin to consider the cost-effectiveness of alternative management actions is particularly encouraging. Nevertheless, our study reveals considerable limitations in our understanding and provides insight into those aspects of malleefowl dynamics that would most benefit from future research. Most importantly, we lack quantitative data on the demographics of adult malleefowl, and our understanding of two key threatening processes, fire and grazing, is poor.

\section{Acknowledgements}

This research forms part of the Cliffs Natural ResourcesWestern Australian Department of Environment and Conservation Land Management Project. We thank I. Harris, M. Bamford and P. West for useful discussions and access to their unpublished data. We appreciate the comments of two anonymous reviewers. M. Bode was funded by the Australian Research Council Centre of Excellence for Environmental Decisions.

\section{References}

Ayers, D., Nash, S. \& Baggett, K. (1996) Threatened Species of Western New South Wales. New South Wales National Parks and Wildlife Service, Sydney, Australia.

Beissinger, S. \& McCullough, D.R. (2002) Population Viability Analysis. University of Chicago Press, Chicago, USA.

Benshemesh, J. (1992) The conservation ecology of malleefowl, with particular regard to fire. $\mathrm{PhD}$ thesis, Monash University, Victoria, Australia.

Benshemesh, J. (1994) Malleefowl (Leipoa ocellata). Flora and Fauna Guarantee Action Statement. Department of Sustainability and Environment, Melbourne, Australia.

Benshemesh, J. (1997) Proceedings of the Third International Megapode Symposium, Nhill, Australia. Nationaal Natuurhistorisch Museum, Leiden, The Netherlands.

Benshemesh, J. (2004) Monitoring malleefowl: options, problems and solutions. In Katanning National Malleefowl Forum. National Malleefowl Recovery Team, Katanning, Australia.

Benshemesh, J. (2007) National Recovery Plan for Malleefowl Leipoa ocellata. Department of Environment and Heritage, South Australia, Canberra, Australia.

Benshemesh, J. (2008) National Recovery Plan for Malleefowl Leipoa ocellata. Department of Environment and Heritage, South Australia, Canberra, Australia.

Benshemesh, J., Barker, R. \& MacFarlane, R. (2007) Trend Analysis of Malleefowl Monitoring Data. National Malleefowl Monitoring, Population Assessment and Conservation Action Project, Bundoora, Australia.

BirdLife International (2008) Leipoa ocellata. In IUCN Red List of Threatened Species v. 2010.4. Http://www.iucnredlist.org [accessed 4 June 2011].

BirdLife International (2010) Species Factsheet: Leipoa ocellata. Http://www.birdlife.org [accessed 23 September 2010].

Воотн, D.T. (1987) Home range and hatching success of malleefowl, Leipoa ocellata Gould (Megapodiidae), in murray mallee near Renmark, S.A.. Australian Wildlife Research, 14, 95-104.

Brereton, R., Bennett, S. \& Mansergh, I. (1995) Enhanced greenhouse climate change and its potential effect on selected fauna of south-eastern Australia: a trend analysis. Biological Conservation, 72, 339-354.

BrickillL, J. (1987) Breeding success of malleefowl Leipoa ocellata in central New South Wales. Emu, 87, 42-45.

Buckley, Y.M., Bolker, B.M. \& Rees, M. (2007) Disturbance, invasion and re-invasion: managing the weed-shaped hole in disturbed ecosystems. Ecology Letters, 10, 809-817.

Buckley, Y.M., Briese, D.T. \& Rees, M. (2003) Demography and management of the invasive plant species Hypericum perforatum. II. Construction and use of an individual-based model to predict population dynamics and the effects of management strategies. Journal of Applied Ecology, 40, 494-507.

Caswell, H. (2001) Matrix Population Models. Construction, Anal$y$ sis and Interpretation. Sinauer Associates, Sunderland, USA.

Chisholm, R. \& TAylor, R. (2010) Body size and extinction risk in Australian mammals: an information-theoretic approach. Austral Ecology, 35, 616-623.

Coombes, C.J., Dehane, R. \& Wilson, A.L. (2007) Post-release survival of captive-reared malleefowl in western New South Wales. In Proceedings of the Katanning National Malleefowl 
Forum, 2007 (ed. S.J.J.F. Davies). National Malleefowl Recovery Team, Katanning, Australia.

Department of Agriculture (2010) Legislative Status of Foxes and Their Control. Department of Agriculture, Fisheries and Forestry, Canberra, Australia.

Department of the Environment, Water, Heritage and the Arts (2010) Leipoa ocellata. In Species Profile and Threats Database. Department of the Environment, Water, Heritage and the Arts, Canberra, Australia.

Frith, H.J. (1959) Breeding of the malleefowl, Leipoa ocellata Gould (Megapodiidae). CSIRO Wildlife Research, 4, 31-60.

Frith, H.J. (1962) Conservation of the malleefowl, Leipoa ocellata Gould (Megapodiidae). CSIRO Wildlife Research, 7, 33-49.

Gillespie, J. (2007) Captive breeding and management of malleefowl (Leipoa ocellata) at Western Plains Zoo, 1989 to present. In Proceedings of the Katanning National Malleefowl Forum, 2007 (ed. S.J.J.F. Davies), National Malleefowl Recovery Team, Katanning, Australia.

Glen, A.S. \& Dickman, C.R. (2005) Complex interactions among mammalian carnivores in Australia, and their implications for wildlife research. Biological Reviews, 80, 387-401.

Gotelli, N.J. (1991) Metapopulation models, the rescue effect, the propagule rain, and the core-satellite hypothesis. The American Naturalist, 138, 768-776.

Kortner, G. \& Watson, P. (2005) The immediate impact of 1080 aerial baiting to control wild dogs on a spotted-tail quoll population. Wildlife Research, 32, 673-680.

McCutcheon, P. (2009) Abandonment of bird program divides wildlife experts. In 7:30 Report. ABC News, Sydney, Australia.

Mellor, J.W. (1911) Malleefowl on Kangaroo Island. Emu, 11, 35-37.

North, A.J. (1917) The birds of Coolabah and Brewarrina, northwestern New South Wales. Records of the Australian Museum, 11, 57-59.

PARSons, B. (2008) Contraction in the range of malleefowl (Leipoa ocellata) in Western Australia: a comparative assessment using presence-only and presence-absence datasets. Emu, 108, 221-231.

PARSONS, B. (2009) Using community observations to predict the occurrence of malleefowl (Leipoa ocellata) in the Western Australian wheatbelt. Biological Conservation, 142, 364-374.

Priddel, D. \& Wheeler, R. (1989) Survival of malleefowl Leipoa occelata chicks in the absence of ground predators. Emu, 90, $81-87$.

Priddel, D. \& Wheeler, R. (1994) Mortality of captive-raised malleefowl, Leipoa ocellata, released into a mallee remnant within the wheat-belt of New South Wales. Wildlife Research, 21, 543-551.

Priddel, D. \& Wheeler, R. (1996) Effect of age at release on the susceptibility of captive-reared malleefowl Leipoa ocellata to predation by the introduced fox Vulpes vulpes. Emu, 96, 32-41.

Priddel, D. \& Wheeler, R. (1997) Efficacy of fox control in reducing the mortality of released captive-reared malleefowl, Leipoa ocellata. Wildife Research, 24, 469-482.

Priddel, D. \& Wheeler, R. (2003) Nesting activity and demography of an isolated population of malleefowl (Leipoa ocellata). Wildlife Research, 30, 451-464.
Priddel, D. \& Wheeler, R. (2005) Fecundity, egg size and the influence of rainfall in an isolated population of malleefowl (Leipoa ocellata). Wildlife Research, 32, 639-648.

Pulliam, H.R. (1988) Sources, sinks and population regulation. The American Naturalist, 135, 652-661.

Pullin, A.S. \& KNight, T.M. (2009) Doing more good than harm building an evidence-base for conservation and environmental management. Biological Conservation, 142, 931-934.

Runge, M.C., Converse, S.J. \& Lyons, J.E. (2011) Which uncertainty? Using expert elicitation and expected value of information to design an adaptive program. Biological Conservation, 4, 1214-1223.

Sullivan, T.L., Williams, A.F., Messmer, T.A., Hellinga, L.A. \& KYRYCHENKO, S.Y. (2004) Effectiveness of temporary warning signs in reducing deer-vehicle collisions during mule deer migrations. Wildlife Society Bulletin, 32, 907-915.

VAN DER WAAG, J. (2007) How to support the recruitment of malleefowl Leipoa ocellata in small remnants: an overview of project findings. In Proceedings of the Katanning National Malleefowl Forum, 2007 (ed. S.J.J.F. Davies). National Malleefowl Recovery Team, Katanning, Australia.

Warburton, B. \& Cullen, R. (1995) Cost-effectiveness of Different Possum Control Methods. Department of Conservation, Wellington, New Zealand.

Weathers, W.W., Weathers, D.L. \& Seymour, R.S. (1990) Polygyny and reproductive effort in the malleefowl Leipoa ocellata. Emu, 90, 1-6.

Wheeler, R. \& Priddel, D. (2009) The impact of introduced predators on two threatened prey species: a case study from western New South Wales. Ecological Management and Restoration, 10, S117-S123.

WoINARSKI, J.C.Z. (1989) Broombush harvesting in south-eastern Australia. In Mediterranean Landscapes in Australia: Mallee Ecosystems and Their Management (eds J.C. Noble \& R.A. Bradstock), pp. 362-378. CSIRO, Melbourne, Australia.

\section{Appendix}

The appendix for this article is available online at http:// journals.cambridge.org

\section{Biographical sketches}

Michael Bode is an Australian Research Council Postdoctoral Fellow at the School of Botany in the University of Melbourne. He is broadly interested in optimal environmental management based on a judicious application of methods from decision theory. KARL BRENNAN is an ecologist for the Western Australian Department of Environment \& Conservation, with a broad range of interests including how mathematical models and return on investment analyses can help guide practical conservation actions. 